\title{
Molecular Origin of the Odd-Even Effect of Macroscopic Properties of $n$-Alkanethiolate Self-Assembled Monolayers: Bulk or Interface?
}

\author{
Fadwa Ben Amara ${ }^{\ddagger}$ Eric R. Dionne $\ddagger$ Sahar Kassir, Christian Pellerin*, and Antonella Badia* \\ Département de chimie, FRQNT Quebec Centre for Advanced Materials, Université de Montréal, C.P. 6128, succursale \\ Centre-ville, Montréal, QC H3C 3J7 CANADA
}

\begin{abstract}
Elucidating the influence of the monolayer interface versus bulk on the macroscopic properties (e.g., surface hydrophobicity, charge transport, and electron transfer) of organic self-assembled monolayers (SAMs) chemically anchored to metal surfaces is a challenge. This article reports the characterization of prototypical SAMs of $n$-alkanethiolates on gold $\left(\mathrm{CH}_{3}\left(\mathrm{CH}_{2}\right)_{n} \mathrm{SAu}, n=\right.$ 6-19) at the macroscopic scale by electrochemical impedance spectroscopy and contact angle goniometry, and at the molecular level, by infrared reflection absorption spectroscopy. The SAM capacitance, dielectric constant, and surface hydrophobicity exhibit dependencies on both the length $(n)$ and parity ( $n_{\text {odd }}$ or $\left.n_{\text {even }}\right)$ of the polymethylene chain. The peak positions of the $\mathrm{CH}_{2}$ stretching modes indicate a progressive increase in the chain conformational order with increasing $n$ between $n=6$ and 16 . SAMs of $n_{\text {odd }}$ have a greater

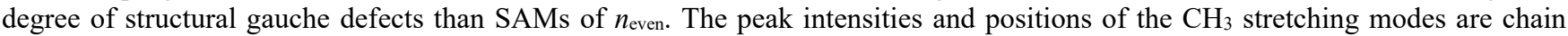
length independent but show an odd-even alternation of the spatial orientation of the terminal $\mathrm{CH}_{3}$. The correlations between the different data trends establish that the chain length dependencies of the dielectric constant and surface hydrophobicity originate from changes in the polymethylene chain conformation (bulk), while the odd-even variation arises primarily from a difference in the chemical composition of the interface related to the terminal group orientation. These findings provide new physical insights into the structure-property relation of SAMs for the design of ultrathin film dielectrics as well as the understanding of stereostructural effects on the electrical characteristics of tunnel junctions.
\end{abstract}

\section{Introduction}

Organic self-assembled monolayers (SAMs) formed on semiconductor, metal or metal oxide surfaces are an integral component of molecular electronic devices, for electrical insulation, charge storage, and charge transport. ${ }^{1-3}$ A recent series of investigations of the charge transport properties of large-area, solidstate tunnel junctions comprised of SAMs of the redox-active ferrocenylalkanethiolates $\left(\mathrm{Fc}\left(\mathrm{CH}_{2}\right)_{n} \mathrm{~S}\right)^{4-5}$ or insulating $n$-alkanethiolates $\left(\mathrm{CH}_{3}\left(\mathrm{CH}_{2}\right)_{n} \mathrm{~S}\right)^{6-9}$ have sparked renewed interest in the odd-even (or parity) effect, ${ }^{10-23}$ which refers to the alternation of a material's structure and/or property due to an odd or even number of repeat units in the molecule. Parity effects in solid condensed phases often arise from differences in intermolecular interactions and molecular packing. A classic example is the odd-even variation of the melting points of solids of oddand even-numbered $n$-alkanes and $n$-alkyl carboxylic acids. ${ }^{24-25}$ SAM properties exhibiting parity effects include surface wettability, friction, and electron transfer rate. ${ }^{26}$ Charge tunneling across metal/SAM/metal junctions is particularly sensitive to the SAM structure. The presence of an odd versus even number of methylenes ( $n_{\text {odd }} / \mathrm{SAM}^{\text {odd }}$ or $n_{\text {even }} / \mathrm{SAM}^{\text {even }}$ ) in the polymethylene chain determines how the organothiolate molecules organize at the supramolecular level and how they couple to the electrodes, which in turn influence the electronic structure and tunnel current density of the junction. $5,15,23$

In $\mathrm{Fc}\left(\mathrm{CH}_{2}\right)_{n} \mathrm{~S}$ SAMs chemisorbed to gold or silver, the parity of the polymethylene chain influences the tilt angle of the terminal ferrocene unit with respect to the surface normal; there is a difference of 5-6 $6^{\circ}$ between $n_{\text {odd }}$ and $n_{\text {even }}$ for $n=6-15 .^{5}$ This odd-even effect in the ferrocene tilt angle leads to a small parity effect in the surface dipole, which in turn causes odd-even effects in the work function and HOMO onset energy of the
$\mathrm{Fc}\left(\mathrm{CH}_{2}\right)_{n} \mathrm{~S}$-functionalized metal surface. ${ }^{5,23}$ These differences in the electronic properties of the SAM alter the shape of the tunneling barrier and lead to an odd-even modulation of the electron tunneling rate across the junction diode in the on state. ${ }^{4-}$ ${ }^{5}$ A smaller ferrocene tilt angle also reduces the steric hindrance and maximizes the chain-chain van der Waals interactions. The computed molecule packing energy is $1.7 \pm 2.9 \mathrm{~kJ} \mathrm{~mol}^{-1}$ lower for $\mathrm{SAM}^{\text {even }}$ (smaller tilt angle) than $\mathrm{SAM}^{\text {odd }}$ (larger tilt angle) on gold. ${ }^{4}{ }^{13}$ Such a small difference in the molecular packing seemingly impacts the performance of devices of which the $\mathrm{SAM}$ is the active component. Specifically, $\mathrm{Fc}\left(\mathrm{CH}_{2}\right)_{n} \mathrm{SAu}$ SAMs of $n_{\text {even }}$ exhibit higher redox potentials and yield a $25 \%$

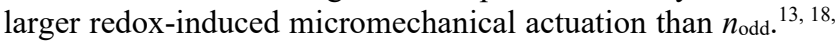
${ }^{21} \mathrm{Fc}\left(\mathrm{CH}_{2}\right)_{n} \mathrm{SAu}$ SAM-based junctions with $n_{\text {even }}$ present lower leakage currents and rectify current, while those with $n_{\text {odd }}$ do not. $^{4-5}$

In the case of junctions formed by SAMs of $\mathrm{CH}_{3}\left(\mathrm{CH}_{2}\right)_{n} \mathrm{~S}$ on gold, whose idealized all-trans extended structure is shown in Figure 1a, SAMs ${ }^{\text {even }}$ yield higher tunneling current densities than their SAM ${ }^{\text {odd }}$ homologues. ${ }^{6}$ The odd-even effect in the tunneling rate is attributed to a difference in the van der Waals interactions at the interface between the SAM and contacting electrode due to the different orientations of the terminal groups in $\mathrm{SAM}^{\text {odd }}$ and $\mathrm{SAM}^{\text {even }}$.,8

Clearly, it is essential to optimize the supramolecular organization of the SAM via the parity of the repeat units in its backbone to harness its full functionality. The perception that due to the large body of published work everything is known about the structural characteristics of SAMs, ${ }^{27}$ including the odd-even effect, ${ }^{26}$ is false. ${ }^{12,14}$ Early structural characterizations of SAMs did not consider the role played by the roughness of the metal 
surface on the SAM order, thereby leading to contradictory results on the existence of a parity effect, ${ }^{12}$ examined a limited number of odd-even homologues, ${ }^{28-30}$ or focused on chains of $n_{\text {odd }}{ }^{31}$. Since the presence of multiple electrodes and interfaces makes it challenging to elucidate the contribution of the inherent supramolecular structure of the organic SAM to the junction properties, recent investigations of the odd-even effect have focused on SAMs chemisorbed to a metal electrode or substrate, probing either the structure of the SAM/ambient interface (near edge X-ray fine structure spectroscopy, ${ }^{5}$ photoemission spectroscopy, ${ }^{23}$ static contact angle, ${ }^{20}$ sum frequency generation spectroscopy, ${ }^{19}$ quartz crystal microbalance ${ }^{22}$ ) or the electronic structure of the SAM/metal interface (ultraviolet photoelectron spectroscopy $)^{5}$. None of these studies however probed directly the conformational order of the polymethylene chains of the SAM; inferences are made in some cases from the odd-even effect observed in the interfacial SAM property (e.g., wettability $)^{20}$ or the spectral signal(s) of the terminal functional groups ${ }^{19,32}$. The structural characterization of both the organic interface and bulk of the SAM is essential for understanding how the former relates to the latter and how each influence the SAM properties.

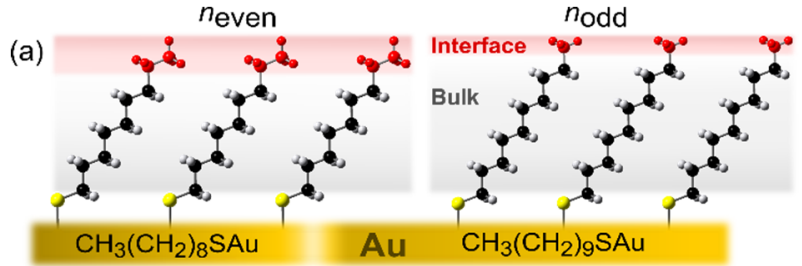

(b)
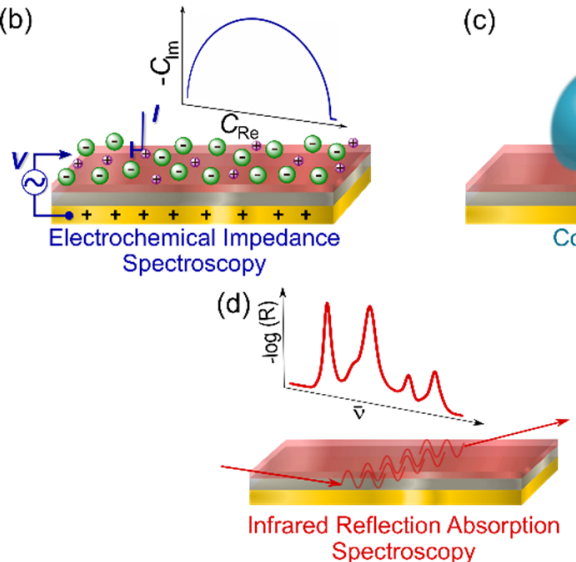

Figure 1. (a) Schematic views of all-trans extended conformers of $\mathrm{CH}_{3}\left(\mathrm{CH}_{2}\right)_{n} \mathrm{~S}$ with an even vs odd number of $\mathrm{CH}_{2}$ repeat units $\left(n_{\text {even }}\right.$ vs $\left.n_{\text {odd }}\right)$ chemisorbed on a gold surface. The Au-S-C bond is fixed at $110^{\circ}$ and the linear backbone of the polymethylene chains is tilted $\sim 20-35^{\circ}$ away from the surface normal (to maximize the chain van der Waals interactions), based on current structural models. ${ }^{26-27,33-34}$ The shaded gray and red slabs denote the bulk and organic interfacial regions of the SAM. The calculated thicknesses of the ethyl-like ( $\left.n_{\text {even }}\right)$ and methyl-like $\left(n_{\text {odd }}\right)$ interfacial layers are $0.185 \mathrm{~nm}$ and $0.055 \mathrm{~nm}$, respectively. ${ }^{6}$ The SAMs were characterized using (b) nonfaradaic electrochemical impedance spectroscopy (capacitance and $\varepsilon$ ), (c) contact angle goniometry (surface hydrophobicity), and (d) infrared reflection absorption spectroscopy (intramolecular organization).
In this regard, we have investigated the chain length dependencies of the dielectric behavior, surface wettability, and supramolecular order of gold-supported SAMs of $\mathrm{CH}_{3}\left(\mathrm{CH}_{2}\right)_{n} \mathrm{~S}$ over a large span of chain lengths $(n=6-19)$. Because parity effects in the electrical properties of SAMs can have significant technological implications, the focus is on charge storage in the interfacial double layer. We sought to identify the respective contributions of the monolayer bulk (polymethylene chain order) and exposed interface (orientational order of the chain termini) to the relative permittivity or dielectric constant $(\varepsilon)$, a key property governing phenomena in electronics, optics, and solid-state physics. SAM characteristics such as the surface coverage density, molecular orientation, polarizability of the terminal group, and bond conjugation have a large effect on the value of $\varepsilon .^{35-36}$ We employed nonfaradaic electrochemical impedance spectroscopy (EIS) to determine the capacitance $(C)$ and $\varepsilon$ (Figure $1 b)$. Water contact angles characterized the surface hydrophobicity of the SAM (Figure 1c). Both dielectric properties as well as the surface hydrophobicity exhibit marked chain length dependent parity effects. Infrared reflection absorption spectroscopy (IRRAS) tracked the evolution of the chain gauche conformational defects and the orientation of the terminal $\mathrm{CH}_{3}$ (Figure 1d) as a function of the molecular length and parity. The IRRAS and contact angle data reveal the structural origins of the odd-even and chain length trends of dielectric properties of the SAM.

\section{Experimental Section}

Details of the chemical reagents used, synthesis and purification of $n$-alkanethiols, preparation of the gold substrates and SAMs, EIS measurements, calculation of the complex capacitance quantity, contact angle goniometry, and IRRAS analyses are given in the Supporting Information.

\section{Results and Discussion}

Interfacial Capacitance of SAMs of $\mathrm{CH}_{3}\left(\mathrm{CH}_{2}\right)_{n} \mathrm{~S}$ on Gold. The electrochemical impedance of polycrystalline gold bead electrodes (Figure S1a of the Supporting Information) modified with SAMs of $\mathrm{CH}_{3}\left(\mathrm{CH}_{2}\right)_{n} \mathrm{~S}$ of $n=6-19$ was measured in $1.0 \mathrm{M}$ $\mathrm{NaClO}_{4(\mathrm{aq})}$ over a frequency span of $1 \mathrm{MHz}$ to $1 \mathrm{~Hz}$ at a structurally non-perturbing potential. ${ }^{37}$ The SAMs are highly impermeable to ions and the SAM-modified gold interfaces exhibit near ideal Helmholtz capacitor behavior (Figure S2 and Table $\mathrm{S} 1)$. Specifically, the complex impedance plane plots comprise nearly vertical lines and the $\mathrm{CH}_{3}\left(\mathrm{CH}_{2}\right)_{n} \mathrm{SAu}$ SAMs present a phase angle of $89^{\circ}$ at low frequency (see Supporting Information for a more detailed discussion) ${ }^{38-39}$ The reactance or opposition of the SAM-modified gold interface to changes in the current flow induced by the ac perturbation of the applied potential, and characterized by the imaginary part $Z_{\mathrm{Im}}$ of the complex impedance $\hat{Z}$, shows dependencies on the length and parity of the chain (Figure S3). These chain length and odd-even effects in $Z_{\mathrm{Im}}$ are in turn reflected in the mathematically-related $C_{\mathrm{Re}}$ (discussed below). No odd-even effect is however observed in the real part $Z_{\mathrm{Re}}$ of the impedance, which is a measure of the resistance of the SAM interface to the dc current flow. 

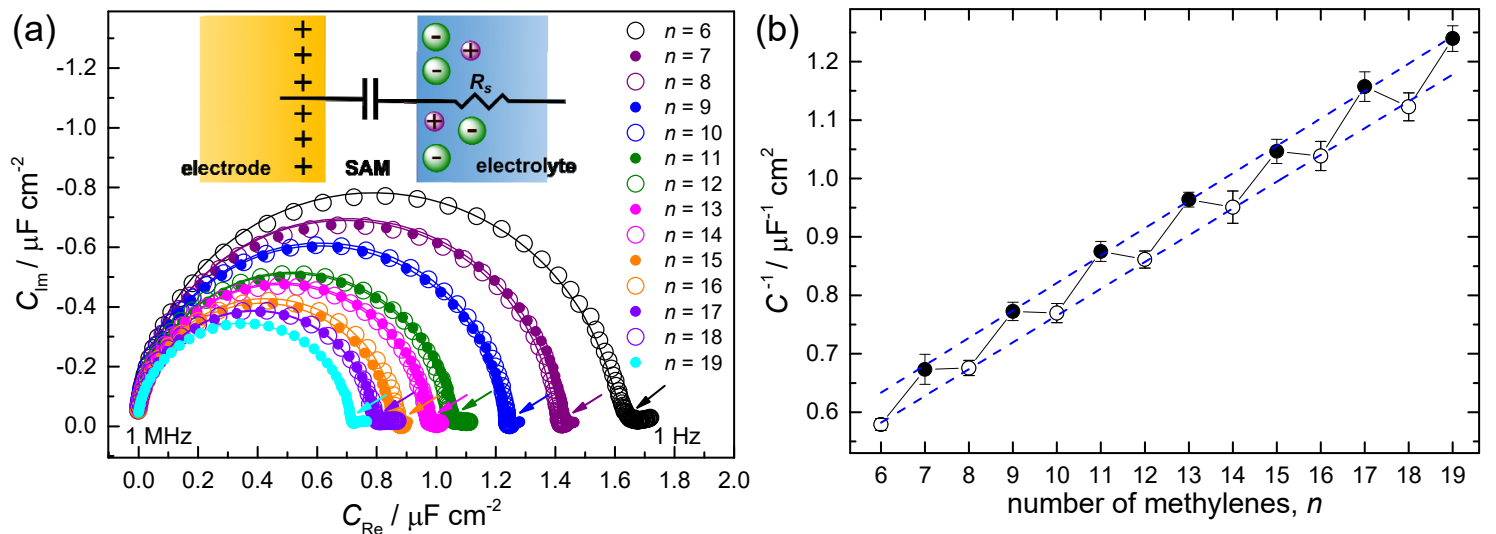

Figure 2. Nonfaradaic EIS of gold electrodes modified with $\mathrm{CH}_{3}\left(\mathrm{CH}_{2}\right)_{n} \mathrm{~S}(n=6-19)$ in $1.0 \mathrm{M} \mathrm{NaClO}_{4(\mathrm{aq})}$ (details in Supporting Information). (a) Complex plane plot of the imaginary $C_{\mathrm{Im}}$ vs real $C_{\mathrm{Re}}$ part of the complex capacitance $\hat{C}$. Symbols are the experimental data and the solid curves represent the fits of the data to the equivalent circuit (shown in inset), consisting of the solution resistance $R_{\mathrm{s}}$ in series with the film capacitance. (b) Plot of the reciprocal of the capacitance $C^{-1}$ as a function of the number of methylene units $n$. Each data point is the mean values of at least 10 electrodes. Error bars represent the $95 \%$ confidence intervals. Dashed blue lines are linear regressions: $r^{2}=0.9993$ for $n_{\text {even }}$ and $r^{2}=0.9986$ for $n_{\text {odd. }}$ Full black lines are guides to the eye.

Shown in Figure $2 \mathrm{a}$ are plots of the imaginary part $C_{\mathrm{Im}}$ versus real part $C_{\mathrm{Re}}$ of the impedance-derived complex capacitance $\hat{C}$ (eq $\mathrm{S} 2-\mathrm{S} 5$ ). $C_{\mathrm{Re}}$ corresponds to the capacitance and $C_{\mathrm{Im}}$ to losses in the form of energy dissipation. ${ }^{40}$ The complex capacitance is better suited to the analysis of insulating dielectric layers than the complex impedance as it highlights the electric charge storage characteristics of the interface rather than its resistive contributions. ${ }^{41-42}$ The complex capacitance plane plots immediately show that (i) the static capacitance (i.e., low-frequency intercept of the semi-circle on the real axis $)^{42}$ decreases with increasing number of methylene units $n$ and (ii) the magnitude of the static capacitance is the same for a SAM of $n_{\text {odd }}$ and its longer chain even neighbor $\left(n_{\text {even }}=n_{\text {odd }}+1\right)$. Bode plots of $C_{\operatorname{Re}}$ (Figure S4) display the same chain length and odd-even dependencies at frequencies $\lesssim 1 \mathrm{kHz}$, where $C_{\mathrm{Re}}$ is independent of frequency.

The impedance data was fit to the series RC-type electrical circuit used to describe the impedance response of ion-blocking SAMs (Figure $2 \mathrm{a}$, inset) ${ }^{18,38,43-44}$ to extract the exact static capacitance value, referred to as $C$ (Figure $\mathrm{S} 4$ and Table S2). The addition of an extra monolayer film resistance $\left(R_{\mathrm{SAM}}\right)$ in parallel to the capacitance does not improve the fits of the data and gives very large values of $\gtrsim 10^{8} \Omega \mathrm{cm}^{2}$, with fitted errors $\gtrsim R_{\mathrm{SAM}}$, for the resistance to ion transport in the SAM across the chain lengths $n=6-19$ investigated (Figure S5), attesting to the highly ionic insulating character of the SAMs ${ }^{39}$ In other words, $R_{\mathrm{SAM}}$ is physically meaningless for the SAMs investigated here, ${ }^{44-45}$ and these can be considered to be effectively free of defects, meaning that current leakage at monolayer defect sites is negligible.

$C^{-1}$ is plotted as a function of $n$ in Figure $2 \mathrm{~b} . \mathrm{CH}_{3}\left(\mathrm{CH}_{2}\right)_{n} \mathrm{SAu}$ $\mathrm{SAMs}^{\text {even }}$ and SAMs ${ }^{\text {odd }}$ exhibit the linear increases of $C^{-1}$ with $n$ expected from a linear increase of the film thickness with chain length (Figure S6). ${ }^{46-48}$ Superimposed on the increase in $C^{-1}$ is the parity effect evident in the complex capacitance plane plots. The capacitances of $n_{\text {odd }} /\left(n_{\text {odd }}+1\right)$ neighbors are statistically indistinguishable, while there is a statistically significant increase in $C^{-1}$ between $n_{\text {even }}$ and $\left(n_{\text {even }}+1\right)$ (99\% confidence level, Table $\mathrm{S} 2$ ). A (quasi-)linear increase in $C^{-1}$ with chain length has been observed in previous electrochemical investigations. ${ }^{31,38,49-50}$ However, none of these studies report an odd-even effect. In some cases, this is simply due to the use of chains of only $n_{\text {odd }}{ }^{31}$,
${ }^{38}$ and in other instances, this can be ascribed to the short-chain odd-even homologues investigated $(n \leq 9)^{49-50}$.

More recently, Jiang and coworkers observed an odd-even chain length dependency in the capacitance of $\mathrm{CH}_{3}\left(\mathrm{CH}_{2}\right)_{n} \mathrm{~S}$ SAMs on silver in large-area junctions with $\mathrm{Ga}_{2} \mathrm{O}_{3} / \mathrm{EGaIn}^{9}{ }^{9}$ The results presented in Figure $2 \mathrm{~b}$ indicate that the parity effect in the film capacitance is not limited to (i) solid-state electrical measurements on SAMs sandwiched between metal contacts, it also manifests itself in wet electrochemical conditions and (ii) SAMs formed on silver, which present significant structural differences to SAMs on gold $^{26-28}$. Both systems present a parity effect in their interfacial charge storage capacity, but a comparison of the measured impedance reveals significant differences in the electrical transport characteristics of the metal-SAMmetal junction versus the ionic insulator properties of the SAM investigated here. We and others ${ }^{38}$ have shown that denselypacked $\mathrm{CH}_{3}\left(\mathrm{CH}_{2}\right)_{n} \mathrm{SAu}$ SAMs effectively behave as blocking layers to ion permeation in electrolyte solution under non-faradaic electrochemical conditions. By contrast, SAM-based junctions (e.g., Ag-SAM-Ga $\mathrm{O}_{3} / \mathrm{EGaIn}^{9}$ and Au-SAMPEDOT:PSS- $\left.\mathrm{Au}^{51}\right)$ present complex impedance responses associated with electronic conduction and that cannot be described by a series RC circuit. $^{42,} 52$ The resistance of the $\mathrm{CH}_{3}\left(\mathrm{CH}_{2}\right)_{n} \mathrm{SAg}$ SAM to electron transport across the tunnel junction increases with $n$ and is $\sim 10^{3}(n=16)$ to $\sim 10^{8}(n=6)$ lower in magnitude (Supporting Information of ref 9) than the resistance to ion transport measured herein for $\mathrm{CH}_{3}\left(\mathrm{CH}_{2}\right)_{n} \mathrm{SAu}$ SAMs. The charge transport resistance of the $\mathrm{CH}_{3}\left(\mathrm{CH}_{2}\right)_{n} \mathrm{SAg}$ SAM also exhibits an odd-even effect that follows the electron tunneling current density $-n_{\text {odd }}$ of lower charge transport resistance yields a larger tunneling current density than $n_{\text {odd }}-1$ of higher charge transfer resistance. ${ }^{9}$ The ion transport resistance of the insulating $\mathrm{CH}_{3}\left(\mathrm{CH}_{2}\right)_{n} \mathrm{SAu}$ SAMs does not exhibit an apparent dependence on $n$ (Figure S5). While defect-free $\mathrm{CH}_{3}\left(\mathrm{CH}_{2}\right)_{n} \mathrm{~S}$ SAMs block ion transport in the potential stability region, ${ }^{37}$ they allow electron transport because the charge carriers travel through the alkyl chain backbone of the $\mathrm{SAM}^{6}$. The different mechanisms of electron and ion transport within the SAM explain its distinct dielectric response in electrical versus electrochemical environments. 
SAM Dielectric Constant and Odd-Even Effect in the SAM Capacitance. There is no evidence that SAMs of $n_{\text {even }}$ are more leaky or permeable to ions, and therefore exhibit a higher capacitance with respect to their chain length, than SAMs of $n_{\text {odd. }}$ So what is at the origin of the observed parity effect? To answer this question, we look to the linear dependence of $C^{-1}$ on $n .^{31,49-50}$ It is consistent with the behavior of an ideal spherical capacitor (i.e., the electrode is a spherical bead), with the gold surface acting as one of the concentric capacitor shells and the physisorbed ions at the SAM/electrolyte interface as the other shell:

$$
C^{-1}=\frac{d}{r(r+d)} \cdot \frac{1}{4 \pi \varepsilon_{\mathrm{o}} \varepsilon_{\mathrm{SAM}}}
$$

where $r$ is the radius of the gold bead $(r=\sim 1.4 \mathrm{~mm}), d$ is the thickness of the SAM dielectric medium separating the two shells $(1.0 \lesssim d \lesssim 2.5 \mathrm{~nm}), \varepsilon_{\mathrm{SAM}}$ is the relative permittivity or dielectric constant of the SAM, and $\varepsilon_{0}$ is the permittivity of free space $\left(8.85419 \times 10^{-12} \mathrm{~F} \mathrm{~m}^{-1}\right) .{ }^{53}$ Since $r \cong\left(10^{5}-10^{6}\right) \times d, r+d \cong$ $r$, and eq 1 simplifies to eq 2 after rearrangement to give the capacitance expressed per unit area:

$$
C^{-1}=\frac{d}{\varepsilon_{\mathrm{o}} \varepsilon_{\mathrm{SAM}}}
$$

The different linear dependencies in $C^{-1}-n$ between SAM ${ }^{\text {odd }}$ and $\mathrm{SAM}^{\text {even }}$ suggest a difference in their respective dielectric constants. Instead of determining an average value of $\varepsilon$ for $\mathrm{SAM}^{\text {even }}$ and $\mathrm{SAM}^{\text {odd }}$ from the slopes of the $C^{-1}-n$ (or $C^{-1}-d$ ) data, ${ }^{9,} 31,38,54-55$ a value was obtained for each chain length to reveal the structure-related variation of $\varepsilon_{\mathrm{SAM}}$ (Figure $3 \mathrm{a}$ and Table S3). Our calculations of $\varepsilon_{\mathrm{SAM}}$ include the weak odd-even fluctuation of $\sim 0.2 \AA$ in the SAM thickness (Figure S7) from the different orientations of the chain termini (Figure 1a). The impact of the parity effect in $d$ on $\varepsilon$ SAM is relatively small. The mean values of ESAM span 1.92 to 2.38 . Published values of $\varepsilon$ determined by electrochemical or optical methods for $\mathrm{CH}_{3}\left(\mathrm{CH}_{2}\right)_{n} \mathrm{SAu}$ SAMs range from 2.0 to $3.0 .^{31,38,48,50,56}$

First and foremost, the plot of $\varepsilon$ sam versus $n$ indicates that $\varepsilon$ of SAM ${ }^{\text {even }}$ is systematically higher than $\varepsilon$ of SAM ${ }^{\text {odd }}$ over the entire range of chain lengths investigated $(n=6-19)$. A leastsquares polynomial fit of the $\varepsilon$ SAM data to separate the contribution of the chain parity from that of the chain length yields an odd-even variation of 0.16 (Figure S8). An odd-even variation of 0.4 was measured in $\varepsilon$ for analogous $\mathrm{SAMs}$ of $\mathrm{CH}_{3}\left(\mathrm{CH}_{2}\right)_{n} \mathrm{~S}$ on silver in junctions with $\mathrm{Ga}_{2} \mathrm{O}_{3} / \mathrm{EGaIn} .{ }^{9} \mathrm{We}$ also conducted EIS measurements on selected chain lengths $(n=9-12)$ using a different electrolyte, i.e., $1.0 \mathrm{M} \mathrm{NaCH}_{3} \mathrm{SO}_{4(\mathrm{aq})}$, to verify that the dielectric behavior observed in $\mathrm{NaClO}_{4}(\mathrm{aq})$ is not electrolytespecific. Indeed, the odd-even difference of $\varepsilon_{S A M}$ of 0.17 determined in $\mathrm{NaCH}_{3} \mathrm{SO}_{4(\mathrm{aq})}$ (Figure S9) is similar to that obtained in $\mathrm{NaClO}_{4(\mathrm{aq})}$. The symmetric $\mathrm{ClO}_{4}^{-}$anion has a zero-dipole moment, while $\mathrm{CH}_{3} \mathrm{SO}_{4}^{-}$possesses a calculated permanent dipole moment of $\sim 6 \mathrm{D}$ (see Supporting Information). One might expect a stronger interaction of the $\mathrm{CH}_{3} \mathrm{SO}_{4}^{-}$with the terminal groups (surface dipoles) of the SAMs compared to the $\mathrm{ClO}_{4}{ }^{-}$, and hence, a difference in the odd-even capacitances determined in the presence of these two electrolyte anions. The absence of such a difference suggests that the observed parity effect in the capacitance does not originate from differences in the dipole interactions between the electrolyte anions and the terminal groups, whose different orientations in SAMs ${ }^{\text {odd }}$ and
$\mathrm{SAMs}^{\mathrm{even}}$ (Figure 1a) yield different interfacial dipole moments. ${ }^{11,20,57}$

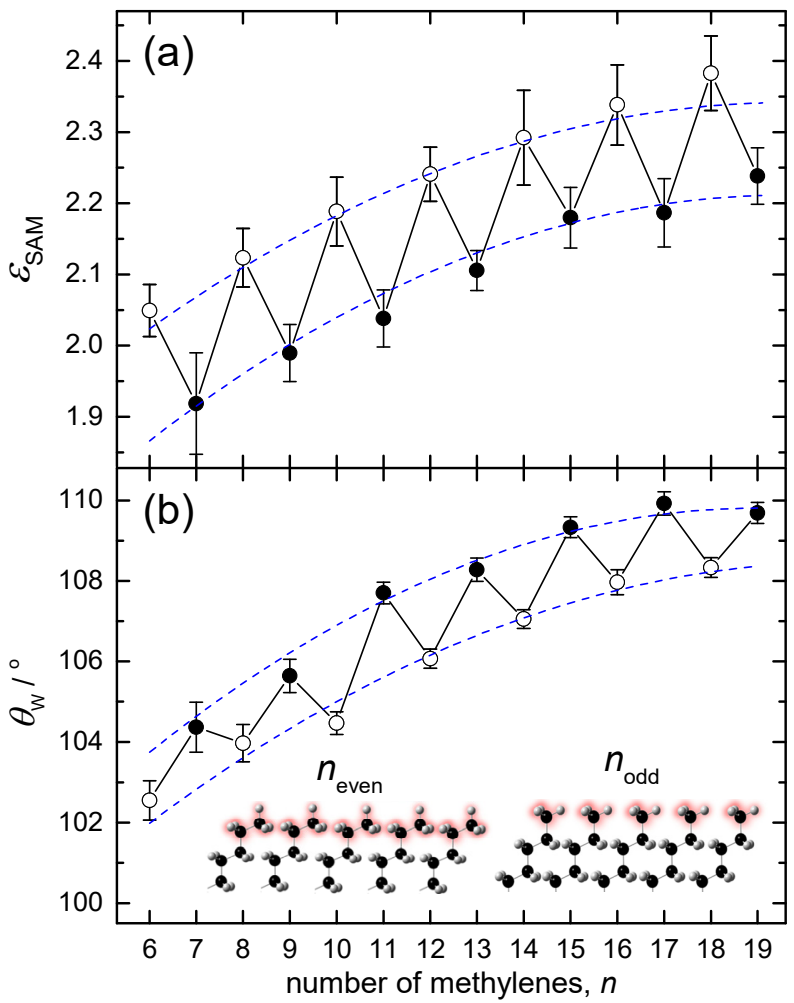

Figure 3. Comparison of the chain length dependencies of (a) SAM dielectric constant ESAM determined from the capacitance using eq 2 and film thickness $d$ calculated assuming a $30^{\circ}$ tilt from the surface normal of all-trans extended alkyl chains and Au-S layer thickness of $0.19 \mathrm{~nm}^{6,48}$ and (b) static contact angle of water $\theta_{\mathrm{w}}$. Error bars represent the $95 \%$ confidence intervals. Solid black lines and dashed blue curves are guides to the eye. Each data point for $\theta_{\mathrm{w}}$ is the mean value of at least 4 different SAMs.

We therefore conclude that the observed odd-even effect in the capacitance originates from an odd-even difference in the SAM dielectric constant. On moving up in $n$ from $n_{\text {even }}$ to $n_{\text {odd, }}$, an increasing film thickness $d$ and a lower \&sam move $C\left(C^{-1}\right)$ in the same direction, i.e., to a lower (higher) value. Moving on from $n_{\text {odd }}$ to $n_{\text {even, }}$ a higher $\varepsilon$ SAM compensates the decreasing effect of an increasing $d$, resulting in little or no change in $C$. Thus, due to the opposing effects of $d$ and $\varepsilon$ on $C$ (eq 2), the capacitance of the longer-chain SAM ${ }^{\text {even }}$ of higher $\varepsilon$ remains (nearly) the same as that of its shorter-chain SAM ${ }^{\text {odd }}$ neighbor of lower $\varepsilon$.

In addition to a parity effect, the plot of $\varepsilon$ sam versus $n$ shows a linear increase between $n=6$ and $15, \Delta \varepsilon=0.033 \pm 0.002$ per $\mathrm{CH}_{2}$ for $n_{\text {even }}$ and $\Delta \varepsilon=0.032 \pm 0.002$ per $\mathrm{CH}_{2}$ for $n_{\text {odd, }}$, followed by a tendency towards limiting values for $n>15$. The dielectric response of saturated hydrocarbons is the same at low and high frequency. ${ }^{35}$ The optical (high-frequency) dielectric constant of $\mathrm{CH}_{3}\left(\mathrm{CH}_{2}\right)_{n} \mathrm{SH}$ liquids increases near-linearly with chain length (Figure S10) due to the higher dielectric constant of $\mathrm{CH}_{2}(\varepsilon=$ 2.164) versus $\mathrm{CH}_{3}(\varepsilon=1.399) .{ }^{58-59}$ The variation of the optical dielectric constant of $\mathrm{CH}_{3}\left(\mathrm{CH}_{2}\right)_{n} \mathrm{SH}$ with chain length, $\Delta \varepsilon \cong$ 0.0035 per $\mathrm{CH}_{2}$, is an order of magnitude smaller than that observed here in the static (low frequency) dielectric constant of the SAM, suggesting that the chain length dependence of EsAM 
does not stem from the number of $\mathrm{CH}_{2}$ 's in the molecule. Moreover, the observed increase in EsAM with $n$ is not due to an increase in the surface coverage density, as predicted by theoretical calculations, ${ }^{35,60-61}$ since the chemisorbed thiolate coverage does not change with chain length or parity over $n=6-19$ (reductive desorption data ${ }^{62-64}$ and high resolution AFM imaging ${ }^{65}$ ).

Odd-Even Effect in the SAM Hydrophobicity. The oddeven chain length dependence of the water contact angle $\left(\theta_{\mathrm{w}}\right)$ on the $\mathrm{CH}_{3}\left(\mathrm{CH}_{2}\right)_{n} \mathrm{SAu}$ SAMs formed on thermally-evaporated gold thin films (Figure S1b) parallels that of $\varepsilon_{\text {sAM }}$ (Figure $3 b$ ), indicating that the same molecular effects are at play. SAMs ${ }^{\text {odd }}$ presenting a "methyl-like" surface exhibit higher contact angles (greater surface hydrophobicity) than SAMs ${ }^{\text {even }}$ of comparable chain length exposing an "ethyl-like" surface (Figure 1a), in agreement with previous reports. ${ }^{16,66}$ The odd-even variation of $\theta_{\mathrm{w}}$ of $1.6^{\circ}$ (Figure $\mathrm{S} 11$ ) is attributable to a difference in the surface free energies of SAMs ${ }^{\text {odd }}\left(\sim 20 \mathrm{~mJ} \mathrm{~m}^{-2}\right)$ and SAMs ${ }^{\text {even }}(\sim 21$ $\left.\mathrm{mJ} \mathrm{m-}{ }^{2}\right)$ related to the terminal group orientations. ${ }^{30}$ On the other hand, the increase of $\theta_{\mathrm{w}}$ from $n=6$ to 16 reflects a change in the bulk phase state of the SAM. ${ }^{16}$ Our IRRAS data, presented in the next section, and that of Porter and coworkers, ${ }^{31}$ indicate an evolution of the $\mathrm{CH}_{3}\left(\mathrm{CH}_{2}\right)_{n} \mathrm{SAu} \mathrm{SAMs}$ from a conformationally-disordered liquid-like phase at short chain lengths to a crystalline-like environment at $n \geq 16$ due to an increase in the cohesive, interchain van der Waals interactions. Additionally, there is a variation in the chain tilt angle (from $22-30^{\circ}$ for $n=6$ to $30-35^{\circ}$ for $n \geq 12$ ) and tilt direction with chain length (grazing incidence $\mathrm{X}$-ray diffraction and molecular dynamics simulations). ${ }^{10,67-68}$ Analogous to $\theta_{\mathrm{w}}$, the observed increase in $\varepsilon_{\text {SAM }}$ as a function of $n$ for $6 \leq n \leq 15$ likely reflects an evolution of the molecular organization and conformational order within the SAM with chain length. The next section addresses these structural characteristics.

Odd-Even Effects in the SAM Molecular Organization. We employed IRRAS to relate the odd-even chain length dependence of the SAM dielectric constant to changes in the polymethylene chain conformation and orientation in the bulk and at the interface of the SAM. IRRAS is performed at a grazing angle of incidence with p-polarized light to selectively enhance the electric field normal to the metal surface. Hence, only the vibrational modes that have a component of their dipole change perpendicular to the surface, and interact strongly with the electric field, are detected. ${ }^{69}$ This metal surface selection rule yields unique information about the spatial geometry of the adsorbate.

Spectra for selected chain lengths of SAMs on gold thin films are presented in Figure S12. These spectra resemble those already published. ${ }^{28-29,31,70}$ The region of interest is that of the $\mathrm{CH}$ stretches between 2800 and $3000 \mathrm{~cm}^{-1}$.

SAM Interface. We begin by assessing the orientation of the terminal $\mathrm{CH}_{3}$ groups (Figure $4 \mathrm{a}$ ). The intensity of the symmetric $\mathrm{CH}_{3}$ stretching mode, $v_{\mathrm{s}}\left(\mathrm{CH}_{3}\right)$, at $2878 \mathrm{~cm}^{-1}$, whose transition dipole moment is colinear with the $\mathrm{C}-\mathrm{CH}_{3}$ axis, ${ }^{28}$ is systematically larger for $n_{\text {odd }}$ than $n_{\text {even }}$. This tendency indicates that the permanent dipole of the $\mathrm{CH}_{3}$ group is oriented more parallel to the surface normal for $n_{\text {odd }}$ than $n_{\text {even }}$ (Figure 1a). The antisymmetric stretching mode, $v_{a}\left(\mathrm{CH}_{3}\right)$, at $2965 \mathrm{~cm}^{-1}$, shows the opposite trend because its transition dipole is perpendicular to the $\mathrm{C}-\mathrm{CH}_{3}$ bond and parallel to the C-C-C plane, ${ }^{28}$ thus corroborating the change in the orientation of the terminal $\mathrm{CH}_{3}$ between
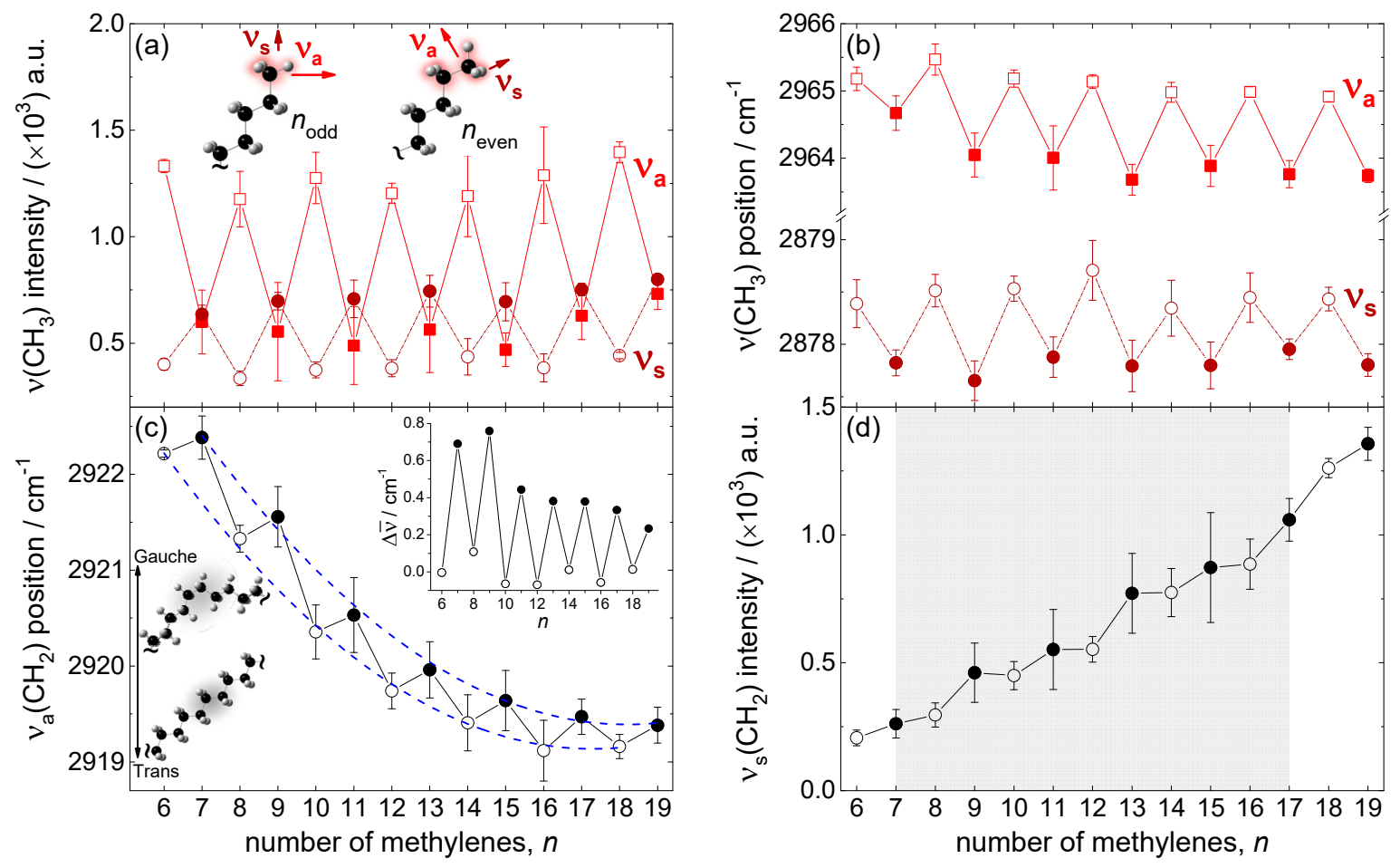

Figure 4. IRRAS of $\mathrm{CH}_{3}\left(\mathrm{CH}_{2}\right)_{n} \mathrm{SAu}$ SAMs. Chain length dependence of (a) $v_{\mathrm{a}}\left(\mathrm{CH}_{3}\right)$ and $v_{\mathrm{s}}\left(\mathrm{CH}_{3}\right)$ peak intensities, (b) v $v_{\mathrm{a}}\left(\mathrm{CH}_{3}\right)$ and $v_{\mathrm{s}}\left(\mathrm{CH}_{3}\right)$ peak positions, (c) $v_{\mathrm{a}}\left(\mathrm{CH}_{2}\right)$ peak position, and (d) $v_{\mathrm{s}}\left(\mathrm{CH}_{2}\right)$ peak intensity. Inset in (c) is a plot of the difference between the experimental positions and those determined from the polynomial fit of the $n_{\text {even }}$ data points. Each data point is the mean value of 5-12 independently prepared SAMs. Error bars represent the 95\% confidence intervals. Dashed blue curves in (c) are second order polynomial fits of the data. Full and dotted lines are guides to the eye. Gray zone in (d) indicates region of odd-even fluctuation. 
$n_{\text {odd }}$ and $n_{\text {even. }}$ The magnitude of this odd-even variation is constant with chain length, analogous to the constant odd-even alternations of $\varepsilon_{\mathrm{SAM}}$ and $\theta_{\mathrm{w}}$ (Figure 3 ). The parity effect in the $v_{\mathrm{s}}\left(\mathrm{CH}_{3}\right)$ and $v_{\mathrm{a}}\left(\mathrm{CH}_{3}\right)$ peak intensities, also observed in previous studies, ${ }^{28,30,71-72}$ is significant because it signals the presence of orientational order at the interfaces of the SAMs prepared herein. Additionally, both the symmetric and antisymmetric $\mathrm{CH}_{3}$ stretching modes exhibit an odd-even oscillation of their peak positions (Figures $4 \mathrm{~b}$ and S12d), a characteristic hereto reported only for $\mathrm{CH}_{3} \mathrm{O}\left(\mathrm{CH}_{2}\right)_{n} \mathrm{SAu}$ SAMs. ${ }^{73}$ There is a $0.7 \pm 0.2$ $\mathrm{cm}^{-1}$ difference in the $v_{\mathrm{s}}\left(\mathrm{CH}_{3}\right)$ peak positions of $n_{\text {even }}$ and $n_{\text {odd }}$ and a $1.1 \pm 0.2 \mathrm{~cm}^{-1}$ difference for $\mathrm{v}_{\mathrm{a}}\left(\mathrm{CH}_{3}\right)$. This odd-even effect could originate from: (i) the different interactions/chemical environments of the $\mathrm{CH}_{3}$ groups in $\mathrm{SAM}^{\text {odd }}$ and $\mathrm{SAM}^{\text {even }}$ and associated intermolecular inductive effect $\left(\mathrm{CH}_{3} \cdots \mathrm{CH}_{3}\right.$ in $n_{\text {odd }}$ and $\mathrm{CH}_{3} \cdots \mathrm{CH}_{2}$ in $\left.n_{\text {even }}\right){ }^{73-74}$ (ii) a difference in the conformational disorder near the chain termini, ${ }^{10,29}$ or (iii) the different interaction potentials of molecules (e.g., water) physisorbed to SAMs of $n_{\text {odd }}$ versus $n_{\text {even }}{ }^{75}$. We cannot at present say which of these three possibilities is the operative mechanism.

Bulk of the SAM. The peak positions of the $\mathrm{CH}_{2}$ stretching modes are qualitative indicators of the degree of conformational disorder, in the form of gauche bond defects, within hydrocarbon-based molecular assemblies, with increasing wavenumber corresponding to increasing disorder. ${ }^{76}$ The symmetric mode, $v_{\mathrm{s}}\left(\mathrm{CH}_{2}\right)$, at $\sim 2850 \mathrm{~cm}^{-1}$ and antisymmetric mode, $v_{\mathrm{a}}\left(\mathrm{CH}_{2}\right)$, at $\sim 2920 \mathrm{~cm}^{-1}$ both show similar chain length dependencies, but we focus on $v_{a}\left(\mathrm{CH}_{2}\right)$ (Figure $4 \mathrm{c}$ ) because it is more sensitive to gauche bond defects than $v_{\mathrm{s}}\left(\mathrm{CH}_{2}\right)$ (Figure S13). ${ }^{31,77}$ There is an exponential-like decrease in wavenumber with increasing $n$, as observed previously by Porter and coworkers for SAMs ${ }^{\text {odd }}{ }^{31}$ The peak positions of 2922.2 and $2853.2 \mathrm{~cm}^{-1}$ for the shortest SAM of $n=6$ point to significant conformational disorder, while the values of 2919.2 and $2850.5 \mathrm{~cm}^{-1}$ for $n=18$ are indicative of polymethylene chains in a predominantly trans-extended conformation for the longest SAMs. ${ }^{31,70,77-78}$ This decrease of the $\mathrm{CH}_{2}$ stretching peak positions with increasing $n$ reflects an ordering of the polymethylene chains and evolution of the SAM phase state from a liquid- to solid-like environment. ${ }^{31}$ Limiting values of the peak positions are attained at $n \geq$ 16 for $v_{\mathrm{a}}\left(\mathrm{CH}_{2}\right)$ and $n \geq 14$ for $v_{\mathrm{s}}\left(\mathrm{CH}_{2}\right)$. Molecular dynamics simulations by Ramin and Jabbarzadeh of the chain length dependence of the structural properties of $\mathrm{CH}_{3}\left(\mathrm{CH}_{2}\right)_{n} \mathrm{~S}$ SAMs on $\mathrm{Au}(111)$ are concordant with the observed trend. ${ }^{10}$ Their calculated percentage of gauche bonds along the molecule decreases from $\sim 12 \%$ for $n=6$ to $\sim 4 \%$ for $n=11$ and remains constant at $\sim 1.5 \%$ for $n>15 .{ }^{10}$ Densely-packed long chain SAMs contain gauche bonds due to thermal motions (concentrated near the chain termini $)^{29}$ and conformational defects at SAM crystal edges from the limited size $(\sim 5-15 \mathrm{~nm})$ of the $\mathrm{CH}_{3}\left(\mathrm{CH}_{2}\right)_{n} \mathrm{SAu}$ domains formed at room temperature ${ }^{67,79-80}$.

Once the degree of intramolecular order stabilizes for chain lengths of $n \geq 16$, the SAM supramolecular structure (including molecular packing defects), and consequently $\varepsilon_{\mathrm{SAM}}$ and $\theta_{\mathrm{w}}$, cease to vary (or vary less) with $n$.

Most importantly, the odd-even effect in the $v_{a}\left(\mathrm{CH}_{2}\right)$ peak positions parallels the one seen in the $C^{-1}-n$ plot (Figure 2b). Polynomial (empirical) fits of the data show that $n_{\text {even }}$ and $n_{\text {odd }}$ exhibit a similar evolution with chain length, but with the peak positions of SAMs ${ }^{\text {odd }}$ shifted to higher wavenumber across the whole series of $\mathrm{CH}_{3}\left(\mathrm{CH}_{2}\right)_{n} \mathrm{~S}$. It is important to note that the reported peak positions are the average of multiple spectra acquired on independently prepared SAMs and that the wavenumber shifts, while small, are telling. The experimentally determined peak positions of SAMs ${ }^{\text {odd }}$ are $\sim 0.7 \mathrm{~cm}^{-1}$ higher for $n$ $=7$ and 9 and $\sim 0.4 \mathrm{~cm}^{-1}$ higher for $n=11-19$ than the "hypothetical" positions (i.e., positions in the absence of an odd-even effect) of the SAMs ${ }^{\text {odd }}$ on the fitted SAMs ${ }^{\text {even }}$ curve (Figure $4 c$, inset), pointing to a greater number of gauche bond conformers in $\mathrm{SAMs}^{\text {odd }}$ versus SAMs ${ }^{\text {even }}$. This odd-even effect in the $\mathrm{va}_{\mathrm{a}}\left(\mathrm{CH}_{2}\right)$ peak position validates the general conclusions of previous theoretical calculations: SAMs ${ }^{\text {even }}$ have a lower percentage of gauche defects than SAMs ${ }^{\text {odd }}$ (i.e., $~ 1.5 \%$ lower for $n=$ 11-14) and are consequently structurally and thermally more stable than SAMs ${ }^{\text {odd }} .{ }^{10}$ A greater conformational order should favor closer packing of the polymethylene chains in SAMs ${ }^{\text {even }}$ and lead to a higher dielectric constant.

We also followed the intensities of the $\mathrm{CH}_{2}$ stretching modes as they provide information on the orientation of the polymethylene chains. The $v_{\mathrm{s}}\left(\mathrm{CH}_{2}\right)$ and $v_{\mathrm{a}}\left(\mathrm{CH}_{2}\right)$ modes are both perpendicular to the main molecular axis. We focus on the $v_{\mathrm{s}}\left(\mathrm{CH}_{2}\right)$ peak as it is less affected by interfering absorption from other vibrational modes than the $v_{a}\left(\mathrm{CH}_{2}\right)$ peak. As expected, the intensity increases with the number of $\mathrm{CH}_{2}$ units in the SAM backbone (Figure 4d). Small local fluctuations of the experimental data are also apparent. Notably, the peak intensity of the shorter $n_{\text {odd }}$ is statistically indistinguishable from that of its longer $n_{\text {even }}$ neighbor for $n=7-16$. Both the percentage of gauche defects and average tilt angle of the polymethylene chains in the SAM influence the $\mathrm{CH}_{2}$ stretching peak intensity for a given $n .{ }^{77,81}$ The greater $\mathrm{v}_{\mathrm{s}}\left(\mathrm{CH}_{2}\right)$ intensity of SAM ${ }^{\text {odd }}$ with respect to its chain length is consistent with the greater orientational disorder denoted by the higher $\mathrm{CH}_{2}$ stretching peak positions (Figures 4c and S13). Gauche conformers partially randomize the orientation of the monolayer $\mathrm{CH}_{2}$ groups relative to the surface (interruption of the all-trans conformation), increasing the intensities of the $\mathrm{CH}_{2}$ stretching modes. ${ }^{77}$ The molecular dynamics simulations that predict a greater conformational disorder for $n_{\text {odd }}$ versus $n_{\text {even }}$ calculate smaller average tilt angles for $n_{\text {odd }}$ compared to $n_{\text {even }}$ (i.e., $\sim 3-4^{\circ}$ smaller for $\left.n=11-14\right) .{ }^{10}$ A more vertical orientation of the chains would accommodate the greater number of gauche bonds found in SAMs ${ }^{\text {odd }}$ by increasing the chain-to-chain spacing (without the need for a lower chemisorbed surface coverage). A smaller chain tilt angle with respect to the surface normal would lower the value of the peak intensity $\left(\mathrm{HCH}\right.$ planes of the $\mathrm{CH}_{2}$ groups are more parallel to the surface when the tilt angle is smaller). ${ }^{31,77}$ We deduce that the contribution to the measured peak intensities of an oddeven difference in the number of gauche defects is greater than that of an odd-even variation in the orientation of the polymethylene chains. The relative intensity of $v_{s}\left(\mathrm{CH}_{2}\right)$ and $\mathrm{v}_{\mathrm{a}}\left(\mathrm{CH}_{2}\right)$ bands provide additional information on the twist angle of the polymethylene chains because their transition dipole moment is parallel and perpendicular to the C-C-C plane, respectively. ${ }^{28}$ The intensity ratio does not vary with $n$ nor with the parity of the chain (Figure S14), in agreement with previous work, ${ }^{28}$ indicating the absence of a chain length dependence of the twist angle.

Bulk or Interface? We employ the chain length trends in the molecular conformational order and orientation within the SAM to discriminate between bulk and interface effects, respectively, to the odd-even effects in the SAM wettability and 
dielectric constant. The length of the $\mathrm{CH}_{3}\left(\mathrm{CH}_{2}\right)_{n} \mathrm{~S}$ molecule affects these macroscopic properties irrespective of the chain parity. The SAM surface becomes more hydrophobic ( $\theta_{\mathrm{w}}$ increases) and $\varepsilon$ SAM increases with $n$ as the degree of conformational order of the polymethylene chains in the monolayer bulk increases. Since SAM $^{\text {odd }}$ has more gauche bond conformers (greater conformational disorder) than $\mathrm{SAM}^{\text {even, }}$ its surface should be more hydrophilic than that of SAM ${ }^{\text {even }}$ of comparable chain length. The opposite behavior is however observed, $\theta_{\mathrm{w}}$ of $n_{\text {odd }}>\theta_{\mathrm{w}}$ of $n_{\text {even, }}$, reaffirming that the parity effect in the SAM hydrophobicity originates from the orientation of the chain termini at the SAM interface (Figure 1a). When the terminal $\mathrm{CH}_{3}$ is oriented parallel to the surface normal $\left(n_{\text {odd }}\right)$, the SAM hydrophobicity and $\theta_{\mathrm{w}}$ increase compared to when it is tilted away from the surface normal $\left(n_{\text {even }}\right)$ due to the lower energy of the methyl-like versus ethyl-like surface. ${ }^{30}$ The bulk and interface of the SAM move $\theta_{\mathrm{w}}$ in opposite directions.

By contrast, the value of $\varepsilon_{\text {SAM }}$ moves in the direction expected from the degree of chain conformational order, i.e., $\varepsilon_{S A M}$ of $\mathrm{SAM}^{\text {odd }}<$ ESAM of SAM ${ }^{\text {even }}$. This does not mean that the parity effect in the SAM dielectric constant is dictated by the bulk. First, the amplitude of the odd-even variation in the $v_{a}\left(\mathrm{CH}_{2}\right)$ peak position decreases with $n$ (Figure $4 \mathrm{c}$, inset), contrary to the parity effect in $\varepsilon$ SAM which remains constant across the entire range of chain lengths investigated. If the odd-even alternation of EsAM stems from the bulk, it should show a reduction in magnitude with $n$. Second, we can estimate the magnitude of the decrease in $\varepsilon$ of SAM $^{\text {odd }}$ that would arise from the difference in the number of chain gauche defects between $\mathrm{SAM}^{\text {even }}$ and $\mathrm{SAM}^{\text {odd }}$ using the chain length dependence of the $\mathrm{v}_{\mathrm{a}}\left(\mathrm{CH}_{2}\right)$ peak position as a metric. If we equate the change in EsAM of 0.312 between $n=6$ and 16 to the corresponding change in the $v_{a}\left(\mathrm{CH}_{2}\right)$ peak position of $3.1 \mathrm{~cm}^{-1}$, then the average shift of 0.53 $\mathrm{cm}^{-1}$ for $n_{\text {odd }}(7-15)$ with respect to its hypothetical even position suggests that $\varepsilon$ SAM would be lowered by an average of 0.049 from its hypothetical value on the trend line of SAMs ${ }^{\text {even }}$. The dielectric constants of SAMs ${ }^{\text {odd }}$ of $n=7-15$ determined from the SAM capacitance are $0.16 \pm 0.02$ lower (i.e., 3.3 fold lower) than the hypothetical even values of $\varepsilon$. The organic interface layer must account for the majority of the experimentally observed parity effect in $\varepsilon_{\text {SAM }}$.

Based on the parallels between the odd-even chain length dependencies of the $\mathrm{CH}_{3}$ and $\mathrm{CH}_{2}$ stretching modes and global SAM dielectric constant $\varepsilon$ SAM, we propose a bilayer stack model (Figure 1a), which assigns separate dielectric constants to the bulk (or interior) of the SAM (cbulk) and to the layer of terminal groups exposed at the SAM/ambient or SAM/electrolyte solution interface ( $\varepsilon_{\text {interface }) .}{ }^{35}$ The increase of $\varepsilon$ with $n$ (odd or even) reflects an increase in $\varepsilon$ bulk due to the progressive ordering of the polymethylene chains and decrease of the gauche defects within the SAM improving the chain packing. The constant $\mathrm{CH}_{3}$ peak intensities and positions (Figure $4 \mathrm{a}, \mathrm{b}$ ) imply that the terminal group orientations and chemical environments in SAMs ${ }^{\text {even }}$ and SAMs ${ }^{\text {odd }}$ are independent of chain length for a given parity. The different water contact angles (Figure $3 b$ ) and friction forces ${ }^{30}$ measured for SAMs ${ }^{\text {even }}$ and SAMs ${ }^{\text {odd }}$ corroborate the distinct chemical nature of the exposed chain termini. These odd-even differences in the chemical composition of the SAM interface influence Einterface, analogous to the SAM hydrophobicity.
Assuming that the $\mathrm{CH}_{2}$ and $\mathrm{CH}_{3}$ groups occupy the same volume, the refractive indices of the individual groups yield an optical dielectric constant of 1.761 for the ethyl-like terminal group (see Supporting Information for the calculation). ${ }^{59}$ The optical dielectric constant of the terminal methyl group is 1.399 , while that of the remaining polymethylene chain is $2.164 .{ }^{59} \mathrm{Cal}-$ culations by Ratner et al. indicate that the dielectric constant of a bilayer film is dominated by the behavior of the layer of the material with the lowest dielectric constant, even if it is much thinner than the accompanying layer of material with higher $\varepsilon .{ }^{35}$ What this means is that the lower Einterface dominates over the higher abulk of the SAM. The ions and water molecules located at the capacitor plate formed by the SAM/electrolyte interface (Figure 2a, inset) are physisorbed onto an ethyl-like surface of higher dielectric constant in the case of the SAM ${ }^{\text {even }}$ versus a methyl-like surface of lower dielectric constant in SAM ${ }^{\text {odd }}$. The estimated difference in the optical dielectric constants of the ethyl and methyl groups of 0.36 is of comparable order of magnitude to the odd-even difference in esAm of 0.16 derived from the capacitance. The calculated difference represents the extreme case of pure methyl and ethyl surfaces. It does not take into account the contribution of the monolayer bulk to $\varepsilon \mathrm{SAM}$ or the orientation of the terminal groups in the SAMs. Even in the idealized representations of Figure 1a, SAM ${ }^{\text {odd }}$ and SAM ${ }^{\text {even }}$ do not present neat methyl and ethyl groups, respectively, due to the imperfect interfacial orientations of the terminal groups in the SAMs. Consequently, the effective dielectric constant of the terminal methyl in SAM ${ }^{\text {odd }}$ would be somewhat larger than the calculated value and that of the ethyl group in SAM ${ }^{\text {even }}$ would be lower, thereby yielding a smaller odd-even difference in $\varepsilon$ SAM. Additionally, the presence of gauche bond conformers in the polymethylene chains, defects at the SAM domain boundaries, and the contribution of the bulk layer (whose chemical

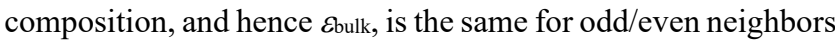
of similar chain lengths) to esAm should also lower the difference measured experimentally between SAM ${ }^{\text {odd }}$ and SAM ${ }^{\text {even }}$. Having ruled out dipole-governed interactions, we ascribe the parity effect in the SAM dielectric constant mainly to the different dielectric constants/chemical compositions of the interfacial layers of the terminal moieties (Einterface).

\section{Conclusions}

We have used surface IR spectroscopy to draw conclusions about the state of the $\mathrm{CH}_{3}\left(\mathrm{CH}_{2}\right)_{n} \mathrm{SAu}$ SAM (dis-)order and associated interface with the number of methylene units (odd and even) in the SAM backbone. This structural information allows us to elucidate the relative contributions of the bulk versus interface of the SAM to the dielectric properties determined by electrochemical impedance spectroscopy.

Odd-Even Chain Length Dependence of the Chain Conformational Order and Orientation. The chain conformational order of the $\mathrm{CH}_{3}\left(\mathrm{CH}_{2}\right)_{n} \mathrm{SAu}$ SAM improves with molecular length and stabilizes at $n \geq 16$, consistent with the findings of Porter et al. ${ }^{31}$ The $\mathrm{CH}_{2}$ peak positions also reveal an unprecedented odd-even difference in the degree of gauche bond defects across the whole series of $\mathrm{CH}_{3}\left(\mathrm{CH}_{2}\right)_{n} \mathrm{~S}$ investigated, with $\mathrm{SAM}^{\text {odd }}$ having more gauche conformers than its shorter $\mathrm{SAM}^{\text {even }}$ neighbor. Previous temperature-dependent currentvoltage and IR spectroscopy measurements on monolayers of polymethylene chains chemically bound to oxide-free silicon indicate that thermally-induced intramolecular disorder decreases the electron transport efficiency along the molecules 
and the measured tunneling current. ${ }^{82}$ The greater number of gauche conformational defects in SAM ${ }^{\text {odd }}$ may therefore contribute to the lower tunneling current densities observed for junctions of $\mathrm{CH}_{3}\left(\mathrm{CH}_{2}\right)_{n} \mathrm{SAu} \mathrm{SAMs}^{\text {odd }}$ with respect to their SAM $^{\text {even }}$ homologues. ${ }^{6}$

The different spatial orientations of the terminal methyl groups in $\mathrm{SAM}^{\text {even }}$ and SAM ${ }^{\text {odd }}$ manifest themselves as an oddeven alternation of the $\mathrm{CH}_{3}$ peak intensities. Additionally, the constant spectral intensities in SAMs ${ }^{\text {even }}$ and in SAMs ${ }^{\text {odd }}$, also reported by Chen et al. down to $n=4,{ }^{71}$ indicate that the terminal group orientations are not affected by the chain length dependent conformational disorder. This chain length independent trend explains why the parity effects observed in the $\mathrm{CH}_{3}\left(\mathrm{CH}_{2}\right)_{n} \mathrm{SAu} \mathrm{SAM}$ dielectric constant, surface hydrophobicity, and in the electron transport rate across $\mathrm{CH}_{3}\left(\mathrm{CH}_{2}\right)_{n} \mathrm{SAu}$ SAM-based junctions ${ }^{6}$, which are all ascribed to an odd-even difference in the terminal group orientation, persist down to short chain lengths. It challenges a common view, derived from the bottom-up organization of tilted, all-trans polymethylene chains, that gauche defects in the chain randomize the spatial orientation of the terminal group, thereby blurring the distinction between the surfaces presented by $\mathrm{CH}_{3}\left(\mathrm{CH}_{2}\right)_{n} \mathrm{~S}$ of $n_{\text {even }}$ and $n_{\text {odd. }}{ }^{20}$ Our data sets imply that disorder in the bulk of the SAM does not preclude the preferential orientation of the chain termini at the surface/interface. This finding has immediate ramifications for the interpretation of the odd-even chain length dependence of SAM interfacial properties, in which the diminution or absence of odd-even fluctuations at shorter chain lengths is ascribed to increased chain conformational defects and the consequent loss of interfacial order. ${ }^{20}$

Capacitance Spectroscopy Directly Reveals Odd-Even Chain Length Differences. The complex impedance response, of the as-prepared SAMs is consistent with ionic insulating behavior. Conversion of the complex impedance to the complex capacitance brings to the fore the charge storage in the interfacial double layer. The capacitance spectra of the insulating $\mathrm{CH}_{3}\left(\mathrm{CH}_{2}\right)_{n} \mathrm{SAu}$ SAMs display outrightly the chain length (expected) and odd-even dependencies of the SAM capacitance. The capacitance of the longer chain $n_{\text {even }}$ is statistically indistinguishable from that of its shorter $n_{\text {odd }}$ neighbor.

Odd-Even Effect in the SAM Dielectric Constant. The SAM dielectric constants derived from the capacitance values using an ideal capacitor model exhibit a chain length dependency and a constant odd-even oscillation of 0.139 , with $\varepsilon$ of $\mathrm{SAM}^{\text {even }}>\varepsilon$ of $\mathrm{SAM}^{\text {odd }}$. The higher dielectric constant of $\mathrm{SAM}^{\text {even }}$ raises its capacitance to the value of its shorter-chain $\mathrm{SAM}^{\text {odd }}$ neighbor of lower dielectric constant. The odd-even chain length dependence of esAm cautions against the use of a single dielectric constant in ellipsometric measurements of SAM thicknesses. ${ }^{31,46-48}$

Orientation-Induced Changes in the Chemical Composition of the SAM Interface are the Origin of the Odd-Even Effect in the SAM Dielectric Constant and Surface Hydrophobicity. The observed odd-even difference in the SAM capacitance is primarily due to differences in the dielectric constants of the ultrathin layers of the terminal moieties exposed at the surfaces of $\mathrm{SAM}^{\text {even }}$ and $\mathrm{SAM}^{\text {odd }}$. More than two decades ago, Gupta and Abbott reported a dramatic $90^{\circ}$ rotation in the macroscopic azimuthal orientations of nematic liquid crystals deposited on SAMs ${ }^{\text {even }}$ versus SAMs ${ }^{\text {odd }}$ of $\mathrm{CH}_{3}\left(\mathrm{CH}_{2}\right)_{n} \mathrm{~S}$ formed on gold surfaces with an anisotropic topography. ${ }^{83}$ No odd-even effect was observed in the liquid crystal anchoring on SAMs on silver, for which the methyl orientation is similar in $\mathrm{SAMs}{ }^{\text {even }}$ and SAMs ${ }^{\text {odd }}{ }^{28}$ Gupta and Abbott thus concluded that the orientation of the terminal groups within the SAMs formed on gold plays an important role in determining the azimuthal orientations of liquid crystals on these surfaces. They hypothesized that if a flexoelectric polarization of the liquid crystals is the cause of the observed parity effect, the different orientations of the methyl groups in SAM ${ }^{\text {even }}$ and SAM ${ }^{\text {odd }}$ must change the dielectric constant of the SAM in contact with the liquid crystals, ${ }^{83}$ just as these alter the surface hydrophobicity. Our results experimentally validate their hypothesis.

In summary, the odd-even effect in the SAM dielectric constant derived from the interfacial capacitance highlights the distinct physicochemical properties that the orientation of the chain termini can impart to the surface/interface. The findings have implications for charge storage at/charge transfer across interfacial molecular assemblies in applications such as gate dielectrics for organic field-effect transistors, molecular tunneling junctions, and capacitive biosensors as well as for theoretical studies on the structure and stability of SAMs.

\section{ASSOCIATED CONTENT}

Supporting Information. Experimental details, synthesis and purification of $n$-alkanethiols, AFM images of the gold surfaces, statistical analyses, calculations, IRRAS spectra, and additional data (PDF). This material is available free of charge via the Internet at http://pubs.acs.org.

\section{AUTHOR INFORMATION}

\section{Corresponding Authors}

*antonella.badia@umontreal.ca

*c.pellerin@umontreal.ca

\section{Author Contributions}

$\$$ These authors (F.B.A. and E.R.D.) contributed equally.

\section{Notes}

The authors declare no competing financial interest.

\section{ACKNOWLEDGMENT}

This research was supported by the Natural Sciences and Engineering Research Council of Canada through grant numbers RGPIN03588-2014 (A.B.), RGPAS-462153-2014 (A.B.), and RGPIN04014-2015 (C.P.). F.B.A. thanks the Tunisian Ministry of Higher Education and Scientific Research for a scholarship. The authors thank Dr. Violeta Toader (FRQNT Quebec Centre for Advanced Materials) for the synthesis and purification of $n$-alkanethiols used in this study.

\section{REFERENCES}

1. Casalini, S.; Bortolotti, C. A.; Leonardi, F.; Biscarini, F., Self-Assembled Monolayers in Organic Electronics. Chem. Soc. Rev. 2017, 46 (1), 40-71.

2. Vilan, A.; Aswal, D.; Cahen, D., Large-Area, Ensemble Molecular Electronics: Motivation and Challenges. Chem. Rev. 2017, 117 (5), 4248-4286.

3. Fabre, B., Functionalization of Oxide-Free Silicon Surfaces with Redox-Active Assemblies. Chem. Rev. 2016, 116 (8), 4808-4849.

4. Nerngchamnong, N.; Yuan, L.; Qi, D.-C.; Li, J.; Thompson, D.; Nijhuis, C. A., The Role of van der Waals Forces in the 
Performance of Molecular Diodes. Nat. Nanotechnol. 2013, 8 (2), 113 118 .

5. Yuan, L.; Thompson, D.; Cao, L.; Nerngchangnong, N.; Nijhuis, C. A., One Carbon Matters: The Origin and Reversal of OddEven Effects in Molecular Diodes with Self-Assembled Monolayers of Ferrocenyl-Alkanethiolates. J. Phys. Chem. C 2015, 119 (31), 1791017919.

6. Baghbanzadeh, M.; Simeone, F. C.; Bowers, C. M.; Liao, K.-C.; Thuo, M.; Baghbanzadeh, M.; Miller, M. S.; Carmichael, T. B.; Whitesides, G. M., Odd-Even Effects in Charge Transport across nAlkanethiolate-Based SAMs. J. Am. Chem. Soc. 2014, 136 (48), 16919-16925.

7. Thuo, M. M.; Reus, W. F.; Nijhuis, C. A.; Barber, J. R.; Kim, C.; Schulz, M. D.; Whitesides, G. M., Odd-Even Effects in Charge Transport across Self-Assembled Monolayers. J. Am. Chem. Soc. 2011, 133 (9), 2962-2975.

8. Chen, J.; Giroux, T. J.; Nguyen, Y.; Kadoma, A. A.; Chang, B. S.; VanVeller, B.; Thuo, M. M., Understanding Interface (OddEven) Effects in Charge Tunneling using a Polished EGaIn Electrode. Phys. Chem. Chem. Phys. 2018, 20 (7), 4864-4878.

9. Jiang, L.; Sangeeth, C. S. S.; Nijhuis, C. A., The Origin of the Odd-Even Effect in the Tunneling Rates across EGaIn Junctions with Self-Assembled Monolayers (SAMs) of n-Alkanethiolates. $J . A m$. Chem. Soc. 2015, 137 (33), 10659-10667.

10. Ramin, L.; Jabbarzadeh, A., Odd-Even Effects on the Structure, Stability, and Phase Transition of Alkanethiol SelfAssembled Monolayers. Langmuir 2011, 27 (16), 9748-9759.

11. Newcomb, L. B.; Tevis, I. D.; Atkinson, M. B. J.; Gathiaka, S. M.; Luna, R. E.; Thuo, M., Odd-Even Effect in the Hydrophobicity of n-Alkanethiolate Self-Assembled Monolayers Depends upon the Roughness of the Substrate and the Orientation of the Terminal Moiety. Langmuir 2014, 30 (40), 11985-11992.

12. Wang, Z.; Chen, J.; Oyola-Reynoso, S.; Thuo, M., The Porter-Whitesides Discrepancy: Revisiting Odd-Even Effects in Wetting Properties of n-Alkanethiolate SAMs. Coatings 2015, 5, 10341055 .

13. Nerngchamnong, N.; Thompson, D.; Cao, L.; Yuan, L.; Jiang, L.; Roemer, M.; Nijhuis, C. A., Nonideal Electrochemical Behavior of Ferrocenyl-Alkanethiolate SAMs Maps the Microenvironment of the Redox Unit. J. Phys. Chem. C 2015, 119 (38), 21978-21991.

14. Chen, J.; Tevis, I.; Gathiaka, S.; Thuo, M., Stereo-Electronic Effects in Tunneling Junctions: Revisiting the Platform. Procedia Eng. 2016, 141, 138-143.

15. Thompson, D.; Nijhuis, C. A., Even the Odd Numbers Help: Failure Modes of SAM-Based Tunnel Junctions Probed via Odd-Even Effects Revealed in Synchrotrons and Supercomputers. Acc. Chem. Res. 2016, 49 (10), 2061-2069.

16. Chen, J.; Chang, B.; Oyola-Reynoso, S.; Wang, Z.; Thuo, M., Quantifying Gauche Defects and Phase Evolution in SelfAssembled Monolayers through Sessile Drops. ACS Omega 2017, 2 (5), 2072-2084.

17. Song, P.; Thompson, D.; Annadata, H. V.; Guerin, S.; Loh, K. P.; Nijhuis, C. A., Supramolecular Structure of the Monolayer Triggers Odd-Even Effects in the Tunneling Rates across Noncovalent Junctions on Graphene. J. Phys. Chem. C 2017, 121 (8), 4172-4180.

18. Feng, Y.; Dionne, E. R.; Toader, V.; Beaudoin, G.; Badia, A., Odd-Even Effects in Electroactive Self-Assembled Monolayers Investigated by Electrochemical Surface Plasmon Resonance and Impedance Spectroscopy. J. Phys. Chem. C 2017, 121 (44), 2462624640.

19. Chen, J.; Liu, J.; Tevis, I. D.; Andino, R. S.; Miller, C. M.; Ziegler, L. D.; Chen, X.; Thuo, M. M., Spectroscopic Evidence for the Origin of Odd-Even Effects in Self-Assembled Monolayers and Effects of Substrate Roughness. Phys. Chem. Chem. Phys. 2017, 19, 6989-6995.

20. Chen, J.; Wang, Z.; Oyola-Reynoso, S.; Thuo, M. M., Properties of Self-Assembled Monolayers Revealed via Inverse Tensiometry. Langmuir 2017, 33 (47), 13451-13467.

21. Dionne, E. R.; Dip, C.; Toader, V.; Badia, A., Micromechanical Redox Actuation by Self-Assembled Monolayers of
Ferrocenylalkanethiolates: Evens Push More Than Odds. J. Am. Chem. Soc. 2018, 140 (32), 10063-10066.

22. Cheng, H.-W.; Wu, Z.-P.; Yan, S.; Li, J.; Shan, S.; Wang, L.; Porter, M. D.; Zhong, C.-J., A Simple Vaporous Probe with AtomicScale Sensitivity to Structural Ordering and Orientation of Molecular Assembly. Chem. Sci. 2019, 10 (29), 7104-7110.

23. Cao, L.; Yuan, L.; Yang, M.; Nerngchamnong, N.; Thompson, D.; Yu, X.; Qi, D.-C.; Nijhuis, C. A., The Supramolecular Structure and van der Waals Interactions Affect the Electronic Structure of Ferrocenyl-Alkanethiolate SAMs on Gold and Silver Electrodes. Nanoscale Adv. 2019, 1 (5), 1991-2002.

24. Pradeilles, J. A.; Zhong, S.; Baglyas, M.; Tarczay, G.; Butts, C. P.; Myers, E. L.; Aggarwal, V. K., Odd-Even Alternations in Helical Propensity of a Homologous Series of Hydrocarbons. Nat. Chem. 2020, $12,475-480$.

25. Yang, K.; Cai, Z.; Jaiswal, A.; Tyagi, M.; Moore, J. S.; Zhang, Y., Dynamic Odd-Even Effect in Liquid n-Alkanes near Their Melting Points. Angew. Chem. Int. Ed. 2016, 55 (45), 14090-14095.

26. Tao, F.; Bernasek, S. L., Understanding Odd-Even Effects in Organic Self-Assembled Monolayers. Chem. Rev. 2007, 107 (5), 1408-1453.

27. Love, J. C.; Estroff, L. A.; Kriebel, J. K.; Nuzzo, R. G.; Whitesides, G. M., Self-Assembled Monolayers of Thiolates on Metals as a Form of Nanotechnology. Chem. Rev. 2005, 105, 1103-1169.

28. Laibinis, P. E.; Whitesides, G. M.; Allara, D. L.; Tao, Y. T.; Parikh, A. N.; Nuzzo, R. G., Comparison of the Structures and Wetting Properties of Self-Assembled Monolayers of n-Alkanethiols on the Coinage Metal Surfaces, Copper, Silver, and Gold. J. Am. Chem. Soc. 1991, 113 (19), 7152-7167.

29. Dubois, L. H.; Zegarski, B. R.; Nuzzo, R. G., Temperature Induced Reconstruction of Model Organic Surfaces. J. Electron Spectrosc. 1990, 54-55, 1143-1152.

30. Wong, S.-S.; Takano, H.; Porter, M. D., Mapping Orientation Differences of Terminal Functional Groups by Friction Force Microscopy. Anal. Chem. 1998, 70, 5209-5212.

31. Porter, M. D.; Bright, T. B.; Allara, D. L.; Chidsey, C. E. D., Spontaneously Organized Molecular Assemblies. 4. Structural Characterization of n-Alkyl thiol Monolayers on Gold by Optical Ellipsometry, Infrared Spectroscopy, and Electrochemistry. J. Am. Chem. Soc. 1987, 109 (12), 3559-3568.

32. Nishi, N.; Hobara, D.; Yamamoto, M.; Kakiuchi, T., ChainLength-Dependent Change in the Structure of Self-Assembled Monolayers of n-Alkanethiols on $\mathrm{Au}(111)$ Probed by BroadBandwidth Sum Frequency Generation Spectroscopy. J. Chem. Phys. 2003, 118 (4), 1904-1911.

33. Ulman, A., Formation and Structure of Self-Assembled Monolayers. Chem. Rev. 1996, 96 (4), 1533-1554.

34. Dubois, L. H.; Nuzzo, R. G., Synthesis, Structure, and Properties of Model Organic Surfaces. Annu. Rev. Phys. Chem. 1992, 43, 437-463.

35. Heitzer, H. M.; Marks, T. J.; Ratner, M. A., Maximizing the Dielectric Response of Molecular Thin Films via Quantum Chemical Design. ACS Nano 2014, 8, 12587-12600.

36. Wang, D.; Fracasso, D.; Nurbawono, A.; Annadata, H. V.; Sangeeth, C. S. S.; Yuan, L.; Nijhuis, C. A., Tuning the Tunneling Rate and Dielectric Response of SAM-Based Junctions via a Single Polarizable Atom. Adv. Mater. 2015, 27 (42), 6689-6695.

37. Boubour, E.; Lennox, R. B., Potential-Induced Defects in nAlkanethiol Self-Assembled Monolayers Monitored by Impedance Spectroscopy. J. Phys. Chem. B 2000, 104 (38), 9004-9010.

38. Boubour, E.; Lennox, R. B., Insulating Properties of SelfAssembled Monolayers Monitored by Impedance Spectroscopy. Langmuir 2000, 16 (9), 4222-4228.

39. Dionne, E. R.; Ben Amara, F.; Badia, A., An Electrochemical Immittance Analysis of the Dielectric Properties of Self-Assembled Monolayers. Can. J. Chem. 2020, doi 10.1139/cjc2020-0005

40. Randriamahazaka, H.; Asaka, K., Electromechanical Analysis by Means of Complex Capacitance of Bucky-Gel Actuators Based on Single-Walled Carbon Nanotubes and an Ionic Liquid. $J$. Phys. Chem. C 2010, 114 (41), 17982-17988. 
41. Góes, M. S.; Rahman, H.; Ryall, J.; Davis, J. J.; Bueno, P. R., A Dielectric Model of Self-Assembled Monolayer Interfaces by Capacitive Spectroscopy. Langmuir 2012, 28 (25), 9689-9699.

42. Orazem, M. E.; Tribollet, B., Electrochemical Impedance Spectroscopy. John Wiley \& Sons, Inc.: Hoboken, N.J., 2008.

43. Janek, R. P.; Fawcett, W. R.; Ulman, A., Impedance Spectroscopy of Self-Assembled Monolayers on $\mathrm{Au}(111)$ : Evidence for Complex Double-Layer Structure in Aqueous $\mathrm{NaClO} 4$ at the Potential of Zero Charge. J. Phys. Chem. B 1997, 101 (42), 8550-8558.

44. Wang, W.; Zhang, S.; Chinwangso, P.; Advincula, R. C.; Lee, T. R., Electric Potential Stability and Ionic Permeability of SAMs on Gold Derived from Bidentate and Tridentate Chelating Alkanethiols. J. Phys. Chem. C 2009, 113 (9), 3717-3725.

45. Olivier, G. K.; Shin, D.; Frechette, J., Factors Governing the Reversible Change in Ionic Permeability of a Low-Density Monolayer. J. Electroanal. Chem. 2010, 639 (1-2), 50-58.

46. Bain, C. D.; Troughton, E. B.; Tao, Y. T.; Evall, J.; Whitesides, G. M.; Nuzzo, R. G., Formation of Monolayer Films by the Spontaneous Assembly of Organic Thiols from Solution onto Gold. J. Am. Chem. Soc. 1989, 111 (1), 321-335.

47. Biebuyck, H. A.; Bain, C. D.; Whitesides, G. M., Comparison of Organic Monolayers on Polycrystalline Gold Spontaneously Assembled from Solutions Containing Dialkyl Disulfides or Alkanethiols. Langmuir 1994, 10 (6), 1825-1831.

48. Shi, J.; Hong, B.; Parikh, A. N.; Collins, R. W.; Allara, D. L., Optical Characterization of Electronic Transitions Arising from the $\mathrm{Au} / \mathrm{S}$ Interface of Self-Assembled n-Alkanethiolate Monolayers. Chem. Phys. Lett. 1995, 246 (1), 90-94.

49. Widrig, C. A.; Chung, C.; Porter, M. D., The Electrochemical Desorption of n-Alkanethiol Monolayers from Polycrystalline $\mathrm{Au}$ and $\mathrm{Ag}$ Electrodes. J. Electroanal. Chem. Interfacial Electrochem. 1991, 310 (1), 335-359.

50. Ramírez, P.; Andreu, R.; Cuesta, Á.; Calzado, C. J.; Calvente, J. J., Determination of the Potential of Zero Charge of Au(111) Modified with Thiol Monolayers. Anal. Chem. 2007, 79 (17), 6473-6479.

51. Akkerman, H. B.; Naber, R. C. G.; Jongbloed, B.; van Hal, P. A.; Blom, P. W. M.; de Leeuw, D. M.; de Boer, B., Electron Tunneling Through Alkanedithiol Self-Assembled Monolayers in Large-Area Molecular Junctions. PNAS 2007, 104 (27), 11161-11166.

52. Bonanos, N.; Pissis, P.; Macdonald, J. R., Impedance Spectroscopy of Dielectrics and Electronic Conductors. In Characterization of Materials, Kaufmann, E. N., Ed. John Wiley \& Sons, Inc.: 2012; pp 1-14.

53. Fujimoto, M., Physics of Classical Electromagnetism. Springer: New York, USA, 1996.

54. Rampi, M. A.; Schueller, O. J. A.; Whitesides, G. M., Alkanethiol Self-Assembled Monolayers as the Dielectric of Capacitors with Nanoscale Thickness. Appl. Phys. Lett. 1998, 72, $1781-1783$.

55. Jiang, L.; Sangeeth, C. S. S.; Yuan, L.; Thompson, D.; Nijhuis, C. A., One-Nanometer Thin Monolayers Remove the Deleterious Effect of Substrate Defects in Molecular Tunnel Junctions. Nano Lett. 2015, 15, 6643-6649.

56. Peterlinz, K. A.; Georgiadis, R., In Situ Kinetics of SelfAssembly by Surface Plasmon Resonance Spectroscopy. Langmuir 1996, 12 (20), 4731-4740.

57. Lee, H. J.; Jamison, A. C.; Lee, T. R., Surface Dipoles: A Growing Body of Evidence Supports Their Impact and Importance. Acc. Chem. Res. 2015, 48 (12), 3007-3015.

58. CRC Handbook of Chemistry and Physics. 100th ed.; CRC Press: 2019.

59. Jung, L. S.; Campbell, C. T.; Chinowsky, T. M.; Mar, M. N.; Yee, S. S., Quantitative Interpretation of the Response of Surface Plasmon Resonance Sensors to Adsorbed Films. Langmuir 1998, 14 (19), 5636-5648.

60. Gala, F.; Zollo, G., Dielectric Properties of Self-Assembled Monolayer Coatings on a (111) Silicon Surface. J. Phys. Chem. C 2015, 119, 7264-7274.
61. Van Dyck, C.; Marks, T. J.; Ratner, M. A., Chain Length Dependence of the Dielectric Constant and Polarizability in Conjugated Organic Thin Films. ACS Nano 2017, 11, 5970-5981.

62. Yang, D. F.; Wilde, C. P.; Morin, M., Electrochemical Desorption and Adsorption of Nonyl Mercaptan at Gold Single Crystal Electrode Surfaces. Langmuir 1996, 12 (26), 6570-6577.

63. Yang, D. F.; Wilde, C. P.; Morin, M., Studies of the Electrochemical Removal and Efficient Re-formation of a Monolayer of Hexadecanethiol Self-Assembled at an $\mathrm{Au}(111)$ Single Crystal in Aqueous Solutions. Langmuir 1997, 13 (2), 243-249.

64. Zhong, C.-J.; Porter, M. D., Fine Structure in the Voltammetric Desorption Curves of Alkanethiolate Monolayers Chemisorbed at Gold. J. Electroanal. Chem. 1997, 425 (1), 147-153.

65. Alves, C. A.; Smith, E. L.; Porter, M. D., Atomic Scale Imaging of Alkanethiolate Monolayers at Gold Surfaces with Atomic Force Microscopy. J. Am. Chem. Soc. 1992, 114 (4), 1222-1227.

66. Wang, Z.; Chen, J.; Gathiaka, S. M.; Oyola-Reynoso, S.; Thuo, M., Effect of Substrate Morphology on the Odd-Even Effect in Hydrophobicity of Self-Assembled Monolayers. Langmuir 2016, 32 (40), 10358-10367.

67. Fenter, P.; Eisenberger, P.; Liang, K. S., Chain-length Dependence of the Structures and Phases of $\mathrm{CH} 3(\mathrm{CH} 2)_{n-1} \mathrm{SH}$ SelfAssembled on Au(111). Phys. Rev. Lett. 1993, 70 (16), 2447-2450.

68. Wang, Y.; Solano Canchaya, J. G.; Dong, W.; Alcamí, M.; Busnengo, H. F.; Martín, F., Chain-Length and Temperature Dependence of Self-Assembled Monolayers of Alkylthiolates on $\mathrm{Au}(111)$ and $\operatorname{Ag}(111)$ Surfaces. J. Phys. Chem. A 2014, 118, 41384146.

69. Hollins, P., Infrared Reflection-Absorption Spectroscopy. In Encyclopedia of Analytical Chemistry, John Wiley \& Sons, Ltd.: 2006; pp 1-17.

70. Nuzzo, R. G.; Dubois, L. H.; Allara, D. L., Fundamental Studies of Microscopic Wetting on Organic Surfaces. 1. Formation and Structural Characterization of a Self-Consistent Series of Polyfunctional Organic Monolayers. J. Am. Chem. Soc. 1990, 112 (2), 558-569.

71. Chen, I. W. P.; Chen, C.-C.; Lin, S.-Y.; Chen, C.-h., Effect of Underpotentially Deposited Adlayers on Sulfur Bonding Schemes of Organothiols Self-Assembled on Polycrystalline Gold: sp or sp3 Hybridization. J. Phys. Chem. B 2004, 108 (45), 17497-17504.

72. Tao, Y.-T., Structural Comparison of Self-Assembled Monolayers of n-Alkanoic Acids on the Surfaces of Silver, Copper, and Aluminum. J. Am. Chem. Soc. 1993, 115, 4350-4358.

73. Wenzl, I.; Yam, C. M.; Barriet, D.; Lee, T. R., Structure and Wettability of Methoxy-Terminated Self-Assembled Monolayers on Gold. Langmuir 2003, 19 (24), 10217-10224.

74. Stole, S. M.; Porter, M. D., In Situ Infrared External Reflection Spectroscopy a a Probe of the Interactions at the LiquidSolid Interface of Long-Chain Alkanethiol Monolayers at Gold. Langmuir 1990, 6 (6), 1199-1202.

75. Engquist, I.; Liedberg, B., D2O Ice on Controlled Wettability Self-Assembled Alkanethiolate Monolayers: Cluster Formation and Substrate-Adsorbate Interaction. J. Phys. Chem. 1996, 100 (51), 20089-20096.

76. Mendelsohn, R.; Flach, C. R., Infrared ReflectionAbsorption Spectrometry of Monolayer Films at the Air-Water Interface. In Handbook of Vibrational Spectroscopy, Chalmers, J. M.; Griffiths, P. R., Eds. John Wiley \& Sons, Ltd.: 2002; Vol. 2, pp 10281041 .

77. Bensebaa, F.; Ellis, T. H.; Badia, A.; Lennox, R. B., Thermal Treatment of n-Alkanethiolate Monolayers on Gold, As Observed by Infrared Spectroscopy. Langmuir 1998, 14, 2361-2367.

78. Nuzzo, R. G.; Korenic, E. M.; Dubois, L. H., Studies of the Temperature-Dependent Phase Behavior of Long Chain n-Alkyl Thiol Monolayers on Gold. J. Chem. Phys. 1990, 93 (1), 767-773.

79. Poirier, G. E., Characterization of Organosulfur Molecular Monolayers on $\mathrm{Au}(111)$ using Scanning Tunneling Microscopy. Chem. Rev. 1997, 97, 1117-1127.

80. Delamarche, E.; Michel, B.; Gerber, C.; Anselmetti, D.; Guentherodt, H. J.; Wolf, H.; Ringsdorf, H., Real-Space Observation 
of Nanoscale Molecular Domains in Self-Assembled Monolayers. Langmuir 1994, 10 (9), 2869-2871.

81. Ulman, A., Thermal Stability of Langmuir-Blodgett and Self-Assembled Films: A Possible Scenario for Order-Disorder Transitions. Adv. Mater. 1991, 3 (6), 298-303.

82. Shpaisman, H.; Seitz, O.; Yaffe, O.; Roodenko, K.; Scheres, L.; Zuilhof, H.; Chabal, Y. J.; Sueyoshi, T.; Kera, S.; Ueno, N.; Vilan, A.; Cahen, D., Structure Matters: Correlating temperature dependent electrical transport through alkyl monolayers with vibrational and photoelectron spectroscopies. Chem. Sci. 2012, 3 (3), 851-862.

83. Gupta, V. K.; Abbott, N. L., Azimuthal Anchoring Transition of Nematic Liquid Crystals on Self-assembled Monolayers Formed from Odd and Even Alkanethiols. Phys. Rev. E 1996, 54 (5), R4540-R4543. 
Insert Table of Contents artwork here

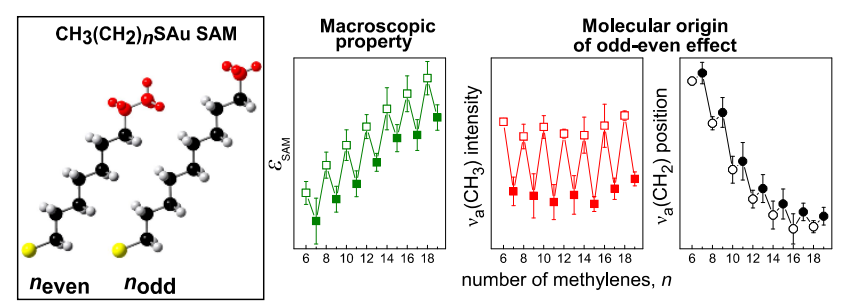

